\title{
ARRONAX, a high intensity cyclotron in Nantes
}

\author{
F. Haddad ${ }^{1}$, N. Michel ${ }^{1}$, A. Guertin ${ }^{1}$, J. Barbet ${ }^{2}$, J.F. Chatal ${ }^{2}$, and J. Martino ${ }^{1}$ \\ 1 SUBATECH, IN2P3-CNRS, Université de Nantes, École des Mines de Nantes, Nantes, France \\ 2 Département de Recherche en Cancérologie, Inserm, Université de Nantes, U601, Nantes, France
}

\begin{abstract}
A cyclotron named ARRONAX is being built in Nantes (France). It is mainly devoted to radiochemistry and nuclear medicine research and will be operational the last quarter of 2008. This machine will accelerate both protons and $\alpha$-particles at high energy (up to $70 \mathrm{MeV}$ ) and high intensity ( 2 simultaneous proton beams with intensity up to $350 \mu \mathrm{A}$ ). In nuclear medicine, these characteristics will allow the cyclotron to produce a large variety of radionuclides on a regular schedule and in sufficient amount to perform clinical trials. A priority list of 12 radioisotopes, which contains isotopes for therapeutic use as well as for PET imaging, has been established by an international scientific committee. In radiochemistry, a vertical pulsed $\alpha$-beam will allow fundamental studies of radiolysis in aqueous media, which is of great interest for radiobiology and for nuclear waste management.
\end{abstract}

\section{Introduction}

During the past decade, a technological revolution has deeply changed the role of radionuclides in medicine especially in oncology.

PET/CT technology with ${ }^{18}$ F-FluoroDeoxyGlucose (FDG) which became clinically available less than 10 years ago, is now accepted as the gold standard in oncology imaging [1]. Moreover some other fluorinated radiopharmaceuticals are currently evaluated [2] and some of them could be approved for a clinical routine use in the coming years.

Radionuclide therapy has been rapidly extended, for the last 10 years, to a large panel of malignant tumors due to the availability of new carriers, including antibodies and peptides, new targeting methods and new radionuclides.

FDG is taken up by any lesion that needs a source of energy for its own metabolism, including inflammatory processes. Such non specific uptake results in a limited diagnostic specificity of FDG-PET. In the future, the use of carriers that are specific for a variety of tumors will allow for specifically targeting cancer cells. This could greatly improve the diagnostic specificity of PET. This new approach, termed immuno-PET, is being evaluated in preclinical studies using different forms of several antibodies. The first results confirmed a higher specificity of immuno-PET as compared to FDG-PET $[3,4]$. However, due to its (too) short physical half-life, fluorine-18 is not appropriate for these carriers that are generally large biological molecules with relatively long (some days) kinetics in the body. Consequently there is a need for innovative positron-emitting radionuclides with half-lives longer than 10 hours.

The future of radionuclide therapy will require increasing injected activity to deliver sufficient irradiation dose to kill radio-resistant solid tumor cells while limiting irradiation of vital organs including liver, lung and kidney. It will be necessary to evaluate, as accurately as possible, the doses absorbed by these organs by a pre-therapeutic dosimetric study, using PET to take advantage of its quantification ability which is superior to that of SPECT (single photon emission tomography). Since most therapeutic radionuclides do not emit positrons, it is interesting to use positron emitters of the same element. They will follow the same metabolic pathways and will allow obtaining the radionuclide biodistribution. Couples of $\beta+/ \beta$ - isotopes of the same element must be available for these studies such as for example ${ }^{124} \mathrm{I} /{ }^{131} \mathrm{I}$ [5].

Finally, another promising approach will be using alpha particle-emitting radionuclides to take advantage of the high LET (linear energy transfer) of alpha particles as compared with that of $\beta$-particles [6].

So, in this context of consequent need for innovative radionuclides, a cyclotron with proton and alpha beams is being built in Nantes, France.

After a description of the accelerator capabilities, we will present the associated facility and the expected list of produced radionuclides.

\section{ARRONAX}

ARRONAX (Accelerator for Research in Radiochemistry and Oncology in Nantes Atlantique) is a high energy (70 MeV) and high intensity ( $750 \mu \mathrm{A}$ proton) cyclotron that will produce a large variety of radionuclides on a regular schedule and in sufficient amount to perform clinical trials. It will also offer the possibility to perform experiments in order to better understand the effect of radiolysis which is always present when dealing with radionuclides in solution as well as radiobiological studies.

\subsection{ARRONAX characteristics}

ARRONAX is being build by IBA. It will turn into operation in the last quarter of 2008 in Nantes (France).

This cyclotron will accelerate both positive ions $(\mathrm{HH}+$, $\mathrm{He}++$ ) and negative ions (H-, D-) up to $70 \mathrm{MeV}$. It is designed to deliver up to $750 \mu \mathrm{A}$ of protons and $35 \mu \mathrm{A}$ of $\alpha$-particles.

- Negative ions will be extracted using a stripper foil. This technique allows extracting the beam within a large range of incident energy. It has been adapted to deliver two 
Table 1. Main characteristics of the ARRONAX cyclotron. (*) the deuteron intensity limit is set for radioprotection reasons.

\begin{tabular}{llll}
\hline & $\begin{array}{l}\text { Accelerated } \\
\text { particles }\end{array}$ & $\begin{array}{l}\text { Energy } \\
\text { Range } \\
(\mathrm{MeV})\end{array}$ & $\begin{array}{l}\text { Intensity } \\
(\mu \mathrm{A})\end{array}$ \\
\hline Protons & $\mathrm{H}-$ & $30-70$ & $<350$ \\
& $\mathrm{HH}+$ & 17.5 & $<50$ \\
Deuterons & $\mathrm{D}-$ & $15-35$ & $50^{*}$ \\
$\alpha$-particles & $\mathrm{He}++$ & 70 & $<35$ \\
\hline
\end{tabular}

beams at the same time. These beams may have different energies and intensities (up to $350 \mu \mathrm{A}$ for protons).

- Positive ions will be extracted using an electromagnetic septum. In this case, beams are extracted at a fixed energy (70 MeV for $\alpha$-particles) and only on one beam output (right side of fig.1).

The capabilities of the ARRONAX cyclotron are summarized in the table 1.

\subsection{The surrounding facility}

The cyclotron will deliver beams in 6 experimental vaults named AX, A1, A2, P1, P2, P3 (see fig. 1). Due to the extraction method which is different for negative and positive ions, protons and deuterons will be available in every experimental vault whereas $\alpha$-particles will be available only in vaults A1, $\mathrm{A} 2$ and $\mathrm{AX}$.

Halls A1, A2, P2 and P3 will be devoted to radionuclide production and will be equipped with an appropriate target system. A rabbit system connected between these vaults and hot cells will limit worker exposition to radiation. These four dedicated vaults will allow flexibility and, together with the high intensity, will ensure availability of radionuclide on a regular schedule.

Vault P1 will be used to perform R\&D on highly intense beam. Indeed, if one wants to use the proton beam delivered by ARRONAX at full intensity and full energy, it will have to deal with $25 \mathrm{~kW}$ on the target. This remains a challenge and works are starting on that subject.

Vault AX, which is the largest, will be devoted to experiments on radiolysis, radiobiology, physics and for highgrade student training. Three beam lines are available in this vault. The $\alpha$-beam can be pulsed. Pulse characteristics are the following: a width equal to $3.3 \mathrm{~ns}$, a variable delay between consecutive (from $1 \mathrm{~ms}$ up to a few seconds), each pulse can contains up to $7 \times 10^{6}$ alphas. In addition for radiolysis and radiobiology experiments a vertical beam is available on one of the lines.

Finally, several laboratories (radiochemistry, biochemistry, cells, radio-labeling, chemical analysis, nuclear metrology...) are available.

\section{The scientific program}

A large part of the ARRONAX beam time will be devoted to the production of radionuclides mainly for medical application. However, taking advantage of the vertical pulsed

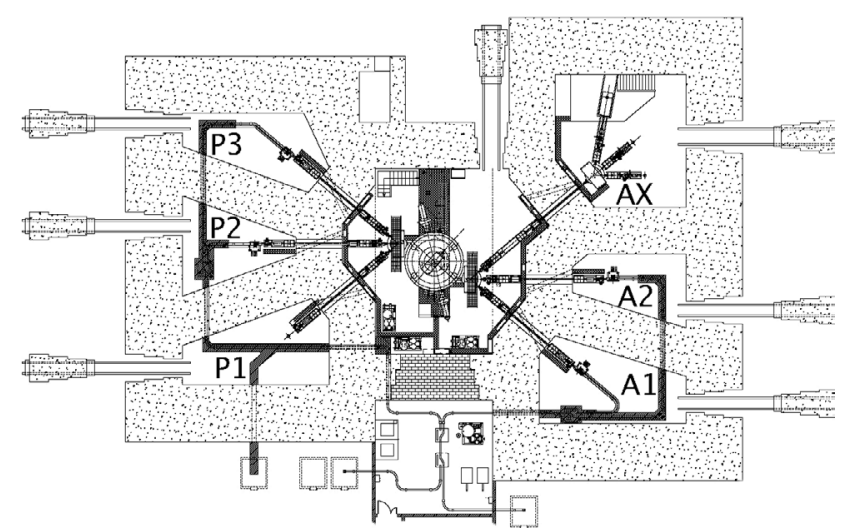

Fig. 1. Schematic view of the ARRONAX facility. This view does not show the different laboratories located around the vaults.

$\alpha$-beam in vault $\mathrm{AX}$, a non negligible beam time will be allowed to radiolysis experiments and radiobiological studies. Fundamental studies of radiolysis in aqueous media are of great interest for radiobiology as well as for nuclear waste management.

In the following section, we will focus on the isotope production. In order to define the most interesting radionuclides to be produced, a priority list has been established by the international scientific committee that has supervised the project since the beginning. This list contains radionuclides for both PET imaging and therapy.

\subsection{Radionuclides for PET imaging}

As presented in the introduction, positron-emitting radionuclides, which are not yet or not readily available, are requested for pre-clinical and clinical applications in oncology as well as for other medical specialties.

Taking advantages of the construction of ARRONAX, we have reinvestigated the periodic table of elements in order to find $\beta^{+}$emitters with longer half lives than ${ }^{18} \mathrm{~F}$ and physical properties adapted to PET imaging. We have also take into account the interest of the scientific community by reviewing the existing literature. Starting from over 500 isotopes, we ended up with 10 radionuclides. They have been separated into two groups based on their interest.

\section{Great interest at short term}

The isotopes for pre-therapeutic use and those available through generators appear among this group.

${ }^{124} \mathrm{I},{ }^{64} \mathrm{Cu},{ }^{86} \mathrm{Y}$ can be used in pre-therapeutic PET dosimetry for an evaluation of tumor and normal organs (liver, kidney, and lung) doses before injection of their $\beta^{-}$emitting counterparts $\left({ }^{131} \mathrm{I},{ }^{67} \mathrm{Cu},{ }^{90} \mathrm{Y}\right)$. It should be noted that ${ }^{86} \mathrm{Y}$ emits non negligible percentages of high energy gamma rays resulting in a relatively high exposure rate and thus requiring some particular radiation safety constraints.

${ }^{82} \mathrm{Rb}$ is an analog of potassium used in cardiology in the United States of America. It is obtained from a ${ }^{82} \mathrm{Sr} /{ }^{82 \mathrm{~g}} \mathrm{Rb}$ generator [7]. Indeed, ${ }^{82} \mathrm{Sr}$ has a long half-life (25.55 days). 
${ }^{68} \mathrm{Ga}$ can be daily available for one year from a generator ${ }^{68} \mathrm{Ge} /{ }^{68} \mathrm{Ga}$ due to the long half-life of ${ }^{68} \mathrm{Ge}$ (270.8 days). However the short half-life of ${ }^{68} \mathrm{Ga}$ (68 minutes) limits its use to the labelling of rapidly diffusible small carriers such as peptides.

\section{Potential interest at middle term}

${ }^{44} \mathrm{Sc}$ may be used to quantify the kinetics and the dosimetry of antibodies when radioimmunotherapy is performed using the innovative radionuclide ${ }^{47} \mathrm{Sc}[8]$.

${ }^{52} \mathrm{Fe}$ has a half-life of $\mathrm{T}_{1 / 2}=8.275 \mathrm{~h}$ and has been used in nuclear medicine mainly for imaging in hematology [9].

${ }^{55} \mathrm{Co},{ }^{76} \mathrm{Br}$, and ${ }^{89} \mathrm{Zr}$ have been identified for immuno-PET due to their high abundance of $\beta^{+}$decay and their relatively long half-life.

\subsection{Radionuclides for therapeutic applications}

Two radionuclides have been identified for $\beta^{-}$therapy ${ }^{67} \mathrm{Cu}$ and ${ }^{47} \mathrm{Sc}$. They can both be produced in cyclotron using the $(p, 2 p)$ reaction and then require high proton beam energy (above $30 \mathrm{MeV}$ ). Their production cross sections are small (few tens of $\mathrm{mb}$ ) and the use of highly intense beam is necessary if ones wants to make them available at a reasonable cost. Radiophysical characteristics of these radionuclides fit well to their use in radionuclide therapy.

Last but not least, the $\alpha$-emitter ${ }^{211}$ At also belongs in our priority list. It seems particularly appropriate for alpha-therapy due to its half-life ( 7.2 hours).

\section{Conclusion}

The high energy (up to $70 \mathrm{MeV}$ ) and high intensity (350 $\mu \mathrm{A}$ for proton and $35 \mu \mathrm{A}$ particle for alpha particle) cyclotron ARRONAX is being built in Nantes (France). It will be devoted to radiochemistry and oncology research and is expected to be in operation in the last quarter of 2008.

A large part of its beam time will be dedicated to the production of radioactive isotopes. In the one hand, PET is most likely to further increase its impact in diagnostic imaging in the future. Positron emitters other than fluorine-18 are clearly needed preferably with slightly longer half-lives $\left({ }^{52} \mathrm{Fe}\right.$, ${ }^{55} \mathrm{Co},{ }^{76} \mathrm{Br}$, $\left.{ }^{89} \mathrm{Zr}\right)$, or produced from generators $\left({ }^{68} \mathrm{Ge} /{ }^{68} \mathrm{Ga}\right.$, $\left.{ }^{82} \mathrm{Sr} /{ }^{82 g} \mathrm{Rb}\right)$, to improve the logistics.

In the other hand, for successful radionuclide therapy, precise calculation of dose distribution, dose planning and dose monitoring are needed. For radioimmunotherapy, quantification of the in vivo distribution of radionuclides as well as their kinetics may be performed efficiently using PET-imaging. Consequently, it is necessary to make available couples of $\beta^{+} / \beta^{-}$isotopes of the same element $\left({ }^{44} \mathrm{Sc} /{ }^{47} \mathrm{Cu}\right.$, $\left.{ }^{64} \mathrm{Cu} /{ }^{67} \mathrm{Cu},{ }^{86} \mathrm{Y},{ }^{124} \mathrm{I}\right)$. Finally, targeted alpha therapy, which takes advantage of the high LET of $\alpha$-particles, is a promising new therapy modality $\left({ }^{211} \mathrm{At}\right)$.

The number of potentially useful radionuclides is relatively limited and the availability of a high-energy, highintensity cyclotron greatly expands this number. Associated with appropriate carriers, these radionuclides will respond to a maximum of unmet clinical needs.

The cyclotron ARRONAX is a project owned by the Regional Council of Pays de la Loire and has been financed by local authorities, the French government and the European Union.

\section{References}

1. G.K. von Schulthess, H.C. Steinert, T.F. Hany. Radiology 422, 238 (2006).

2. O. Couturier, A. Luxen, J.F. Chatal et al., Eur. J. Nucl. Med. Mol. Imaging 31, 1182 (2004)

3. F.T. Lee, A.M. Scott, J. Nucl. Med. 44, 1282 (2003).

4. I. Verel, G.W. Visser, G.A. van Dongen, J. Nucl. Med. 46, Suppl. 1 164S (2005).

5. G. Sgouros, K.S. Kolbert, A. Sheikh, K.S. Pentlow, E.F. Mun, A. Barth et al., J. Nucl. Med. 45, 1366 (2004).

6. M.R. MacDevitt, G. Sgouros, R.D. Finn, J.L. Humm, J.G. Jurcic, S.M. Larson et al., Eur. J. Nucl. Med. 25, 1341 (1998).

7. Special issue on the Strontium-82/Rubidium- 82 generator, Appl. Radiat. Isot. 38 (1987).

8. L.F. Mausner, K.L. Kolsky, V. Joshi, S.C. Srivastava, Appl. Radiat. Isot. 49, 285 (1998).

9. J. Zweit, S. Downey, H. Sharma, Int. J. Rad. Appl. Instrum. 39, 1197 (1988). 